\title{
Weak Formulation of Free-Surface Wave Equations
}

Abstract. An alternative method for deriving water wave dispersion relations and evolution equations is to use a weak formulation. The free-surface displacement $\eta$ is written as an eigenfunction expansion,

$$
\eta=\sum_{n=1}^{\infty} a_{n}(t) E_{n}
$$

where the $a_{n}(t)$ are time-dependent coefficients. For a tank with vertical sides the $E_{n}$ are eigenfunctions of the eigenvalue problem,

$$
\nabla^{2} E+\lambda^{2} E=0, \quad \nabla E \cdot \widehat{\mathbf{n}}=0 \text { on the tank side walls. }
$$

Evolution equations for the $a_{n}(t)$ can be obtained by taking inner products of the linearised equation of motion,

$$
\rho \frac{\partial \mathbf{v}}{\partial t}=-\frac{1}{\rho} \nabla P+\mathbf{F}
$$

with the normal irrotational wave modes. For unforced waves each evolution equation is a simple harmonic oscillator, but the method is most useful when the body force $\mathbf{F}$ is something more exotic than gravity. It can always be represented by a forcing term in the SHM evolution equation, and it is not necessary to assume $\mathbf{F}$ irrotational. Several applications are considered: the Faraday experiment, generation of surface waves by an unsteady magnetic field, and the metal-pad instability in aluminium reduction cells.

Keywords: Waves, weak solution, MHD

\section{Introduction}

Many classical water-wave problems involve irrotational body forces only. The usual method of analysis is to argue that the flow will be irrotational so the solution can be found conveniently in terms of a velocity potential. In magnetohydrodynamics (MHD) however, the Lorentz body force $\mathbf{J} \times \mathbf{B}$ - where $\mathbf{B}$ is magnetic field and $\mathbf{J}$ electric current density - is generally rotational, so the resulting flow will have vorticity, and not admit such a simple description.

The aim of this paper is to describe a method of analysing surface waves where the underlying flow cannot be assumed irrotational. The technique 
is to calculate the flows $\mathbf{v}_{n}, n=1,2 \ldots$ corresponding to the normal irrotational wave modes. Then the inner product of each $\mathbf{v}_{n}$ with the linearised equation of motion provides a set of evolution equations for each of the free surface modes. When gravity is the only body force these equations represent a simple harmonic oscillator; a rotational body force produces an additional forcing term, and the equations can still be solved by simple methods.

Section 2 is devoted to a detailed description of the method, and derivation of the basic equations. In section 3 we look at three applications the Faraday experiment, generation of surface waves by a time-dependent magnetic field, and metal-pad instability in aluminum reduction cells. Our conclusions are summarised in section 4 .

\section{Formulation of Equations}

We consider a tank with vertical walls and an arbitrary (but uniform) horizontal cross-section. It contains incompressible fluid of density $\rho$ to a depth $h$, and we use $V$ to denote the fluid volume, $S_{W}$ the side walls of the tank, and $S_{B}$ its base.

\subsection{The Free Surface Expansion}

To describe the free-surface displacement it is convenient to introduce the functions $E_{n}(x, y), n=1,2, \ldots$, which are the eigenvalues of the problem,

$$
\nabla^{2} E+\lambda^{2} E=0, \quad \nabla E \cdot \widehat{\mathbf{n}}=0 \text { on } S_{W} .
$$

The free surface displacement $\eta$ is expanded in the form

$$
\eta=\sum_{n=1}^{\infty} a_{n}(t) E_{n},
$$

where the $a_{n}$ are functions of time to be determined. The functions $E_{n}$ form an orthogonal sequence:

$$
\int E_{p} E_{q} \mathrm{~d} x \mathrm{~d} y=\delta_{p q}\left\|E_{p}\right\|^{2} ; \quad \int \nabla E_{p} \cdot \nabla E_{q} \mathrm{~d} x \mathrm{~d} y=\delta_{p q} \lambda_{p}^{2}\left\|E_{p}\right\|^{2} .
$$

For containers with simple cross-sections the eigenfunctions can be found explicitly. For example, for a circular tank

$$
e^{\mathrm{i} m \theta} J_{m}\left(\lambda_{m k} r\right), \quad m=0, \pm 1, \pm 2 \ldots, \quad k=1,2, \ldots,
$$


where $m$ is an integer, $J_{m}$ a Bessel function of order $m$, and $\lambda_{m k}$ the $k^{\prime}$ th zero of $J_{m}^{\prime}(a)$. More generally, for an elliptical tank the $E_{n}$ are Mathieu functions, and for the rectangle $0 \leq x \leq a, 0 \leq y \leq b$ they take the form,

$$
\cos (m \pi x / a) \cos (n \pi y / b), \quad m, n, \text { integers. }
$$

We also define the related sequence of harmonic functions, by setting

$$
\phi_{n}(x, y, z)=\frac{\cosh \lambda_{n}(z+h) E_{n}(x, y)}{\lambda_{n} \sinh \lambda_{n} h}=E_{n}(x, y) f_{n}(z) \text { say. }
$$

These functions satisfy

$$
\nabla^{2} \phi_{n}=0, \quad \nabla \phi_{n} \cdot \widehat{\mathbf{n}}=0 \text { on } S_{W} \text { and } S_{B}, \quad\left(\frac{\partial \phi}{\partial z}\right)_{z=0}=E_{n} .
$$

\subsection{Evolution Equations}

The linearised Euler equation of motion is

$$
\rho \frac{\partial \mathbf{v}}{\partial t}=-\nabla P+\mathbf{F}
$$

Here $P=p+\rho g z$, where $p$ is the fluid pressure and $\mathbf{F}$ the body force (excluding gravity). We neglect surface tension, although its inclusion would entail only minor modifications in the absence of dissipation. (A complete treatment of surface tension would have to consider damping due to the movement of the meniscus on the side walls of the container. This is difficult to estimate, although methods have been suggested for example in [3].) On the free surface therefore $p=0$, and the linearised boundary conditions are:

$$
(P)_{z=0}=\rho g \eta, \quad \dot{\eta}=\left(v_{z}\right)_{z=0} .
$$

The inner product of two vector fields $\mathbf{A}, \mathbf{B}$ is defined by setting

$$
\langle\mathbf{A}, \mathbf{B}\rangle=\int_{V} \mathbf{A} \cdot \mathbf{B} \mathrm{d} V
$$

and a convenient way of deriving evolution equations for the free-surface mode coefficients $a_{n}(t)$ is to take the inner product of (8) with each of the test functions $\nabla \phi_{n}$. The first term,

$$
\left\langle\rho \dot{\mathbf{v}}, \nabla \phi_{n}\right\rangle=I_{1} \quad \text { (say) }=\rho \int_{V} \nabla \cdot\left(\phi_{n} \dot{\mathbf{v}}\right) \mathrm{d} V=\rho \int_{S} \phi_{n} \dot{v}_{z} \mathrm{~d} x \mathrm{~d} y,
$$


where $S$ is the free surface $z=0$ (assumed plane for the purposes of this leading-order calculation). We have used the Gauss Divergence Theorem and the tank walls and base, on which $\mathbf{v} \cdot \widehat{\mathbf{n}}=0$, give no contribution to the resulting surface integral. The linearised kinematic free-surface condition

$$
\dot{\eta}=\left(v_{z}\right)_{z=0}
$$

and (6) now give

$$
I_{1}=\rho f_{n}(0) \int_{S} E_{n} \ddot{\eta} \mathrm{d} x \mathrm{~d} y=\frac{\rho}{\lambda_{n} \mathcal{T}_{n}}\left\|E_{n}\right\|^{2} \ddot{a}_{n},
$$

where

$$
\left\|E_{n}\right\|^{2}=\int_{V} E_{n}^{2} \mathrm{~d} V, \quad \mathcal{T}_{n}=\tanh \left(\lambda_{n} h\right) .
$$

The last step uses the expansion (2) for $\eta$ and the orthogonality of the $E_{n}$.

The inner product arising from the pressure term in (8) is

$$
I_{2}=\left\langle\nabla P, \nabla \phi_{n}\right\rangle=\int_{V} \nabla \cdot\left(P \nabla \phi_{n}\right) \mathrm{d} V=\int_{S} P \frac{\partial \phi}{\partial z} \mathrm{~d} x \mathrm{~d} y .
$$

Now using (7), (9) and (2), we obtain

$$
I_{2}=\rho g\left\|E_{n}\right\|^{2} a_{n}(t)
$$

The form of the body force term will depend on the particular application, so for the moment we can do no better than to write it simply as $\frac{\lambda_{n} \mathcal{T}_{n}}{\rho\left\|E_{n}\right\|^{2}}\left\langle\mathbf{F}, \nabla \phi_{n}\right\rangle$.

Combining (10) and (11) we obtain the following evolution equation for the $a_{n}$ :

$$
\ddot{a}_{n}+\Omega_{n}^{2} a_{n}=\frac{\lambda_{n} \mathcal{T}_{n}}{\rho\left\|E_{n}\right\|^{2}}\left\langle\mathbf{F}, \nabla \phi_{n}\right\rangle, \quad \Omega_{n}^{2}=g \lambda_{n} \mathcal{T}_{n},
$$

where the frequencies $\Omega_{n}$ are just the natural gravity-wave frequencies for the tank.

Finally we note that the flow, being irrotational, is determined uniquely by the specification of $v_{z}(x, y)$ on the free surface. It then follows from (2) and (9) that to leading order.

$$
\mathbf{v}=\sum_{n=1}^{\infty} \dot{a}_{n} \nabla \phi_{n} .
$$




\section{Applications}

\subsection{Faraday's Experiment}

When a container of liquid is made to oscillate vertically, standing waves may be observed on the free surface. These were first studied experimentally by Faraday [2], and Benjamin and Ursell [1] showed that the evolution equation for each normal mode is a Mathieu equation. This last result follows almost immediately from our analysis. In a frame of reference oscillating vertically with the tank we must introduce a fictitious body force $g \epsilon \cos \omega t \widehat{\mathbf{z}}$, where $\epsilon$ is the dimensionless amplitude, $\omega$ the frequency of oscillation, and the $z$-direction is vertical. In (12) we simply replace $g$ by $g(1+\epsilon \cos \omega t)$ and obtain the following Mathieu equations:

$$
\ddot{a}_{n}+\Omega_{n}^{2}(1+\epsilon \cos \omega t) a_{n}=0 .
$$

A plane free surface $\eta=0$ is always a solution, but this may be unstable, depending on the values of the parameters $\epsilon$ and $\omega$. The instability regions for the Mathieu functions have been intensively studied (see e.g. [5] ), and can be determined by simple approximate methods when $\epsilon \ll 1$. Figure 1 shows the approximate boundaries in the $(\omega, \epsilon)$-plane for a twodimensional tank of depth $20 \mathrm{~cm}$ and length $50 \mathrm{~cm}$. Wedges of instability arise from the resonance points on the $\epsilon=0$ axis, where $\omega=2 \Omega_{n}$.

The free surface is most easily destabilised if the applied frequency is close to one of the natural frequencies. When the point $(\omega, \epsilon)$ lies in an unstable region the corresponding mode grows exponentially until nonlinear or dissipative effects become important.

\subsection{Waves Due to a Time-Dependent Magnetic Field}

Alternating, rotating or travelling magnetic fields are often used in industry to contain or stir volumes of liquid metal. The field $\mathbf{B}$ induces an electric current $\mathbf{J}$ which interacts with $\mathbf{B}$ to generate a $\mathbf{J} \times \mathbf{B}$ body force, which is generally rotational.

As a model problem we consider the generation of waves in a tank of liquid metal by a time-dependent applied magnetic field. A two-dimensional electrically insulating container of length $\ell$ is filled to a depth $h$ with liquid metal of electrical conductivity $\sigma$. The eigenfunctions and eigenvalues are given by

$$
E_{n}=\cos \left(\frac{n \pi x}{\ell}\right), \quad \lambda_{n}=\frac{n \pi}{\ell} .
$$




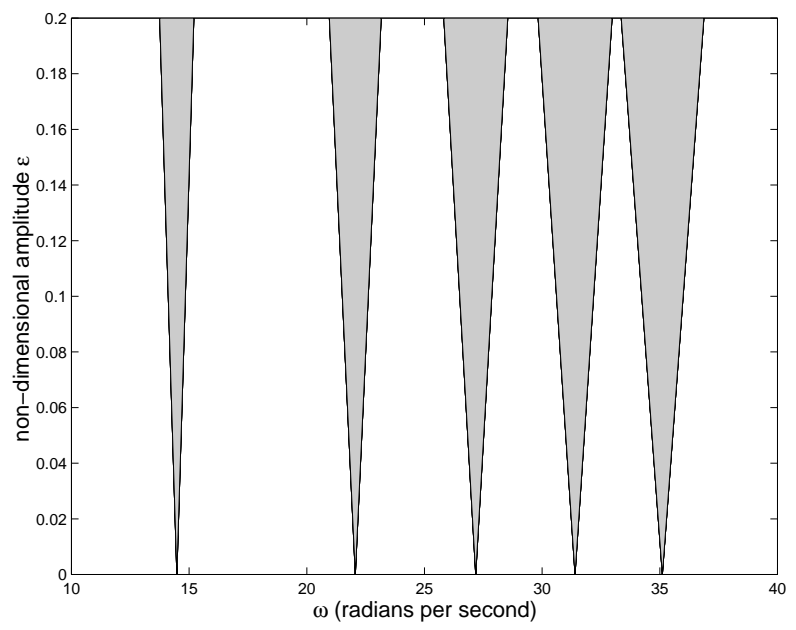

Figure 1. Stability regions for Mathieu's equation in the $(\omega, \epsilon)$-plane. The horizontal axis gives the frequency in radians per second and the vertical axis the non-dimensional amplitude $\epsilon$. The unstable regions are shaded.

We suppose that at time $t=0$ the fluid is at rest. In order to focus attention on the inductive effect of a moving magnetic field we consider an irrotational field which, if stationary, would generate no motion. Specifically we suppose that the field

$$
\mathbf{B}(\mathbf{x}, t)=\nabla Q(x, y, t)
$$

is applied. According to Faraday's law,

$$
\nabla \times \mathbf{E}=-\frac{\partial \mathbf{B}}{\partial t}=-\nabla \dot{Q},
$$

where $\mathbf{E}$ is the electric field. If $S$ is the harmonic function conjugate to $Q$ - i.e. if

$$
\nabla Q=(\nabla S) \times \widehat{\mathbf{z}}=\nabla \times(S \widehat{\mathbf{z}}),
$$

we can write

$$
\nabla \times \mathbf{E}=-\nabla \times(\dot{S} \widehat{\mathbf{z}}) .
$$

It follows that

$$
\mathbf{E}=-\dot{S} \widehat{\mathbf{z}}+\nabla R,
$$

where $R$ is an arbitrary scalar function, and Ohm's law shows that the electric current density $\mathbf{J}$ can be written in the form

$$
\mathbf{J}=\sigma \mathbf{E}=-\sigma \dot{S} \widehat{\mathbf{z}}+\nabla R .
$$


The conditions that $\nabla \cdot \mathbf{J}=0$ and that $\mathbf{J} \cdot \widehat{\mathbf{n}}=0$ on the insulating boundaries imply that $\nabla R=0$, so

$$
\mathbf{J}=-\sigma \dot{S} \widehat{\mathbf{z}}
$$

and the body force is

$$
\mathbf{J} \times \mathbf{B}=-\sigma \dot{S} \nabla S .
$$

We choose a simple potential function for $Q$, writing

$$
Q=\frac{B_{0}}{k} e^{k y} \cos [k x-f(t)], \quad S=-\frac{B_{0}}{k} e^{k y} \sin [k x-f(t)],
$$

where $B_{0}$ represents the magnetic field intensity, $k$ is the wave number of the field, and $f(t)$ a function of time which can be specified in due course. Field lines for one period of this field are shown in figure 2.

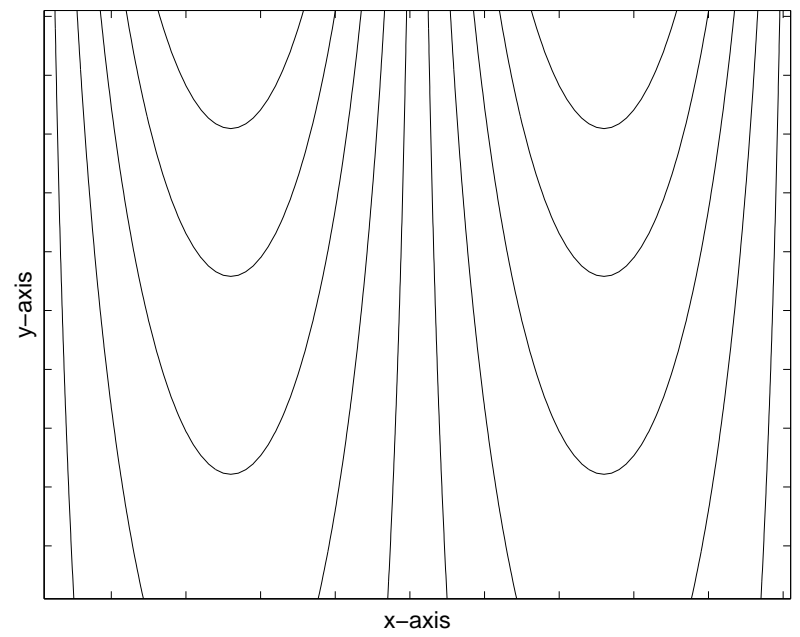

Figure 2. Magnetic field lines

From (14) and (15) it follows that

$$
\mathbf{F}=\frac{\sigma B_{0}^{2} \dot{f}}{2 k}\left[e^{2 k y} \widehat{\mathbf{x}}+\frac{1}{2 k} \nabla\left(e^{2 k y} \sin 2 k \theta\right)\right],
$$

where $\theta=k x-f(t)$, which is clearly a rotational force field. The forcing term on the RHS of the evolution equation (12) can be written in dimen- 
sionless form as

$$
\begin{array}{r}
L \dot{f}^{2} \mathcal{N} \frac{n \pi \tau_{n}}{k^{\prime}\left(4 k^{\prime 2}-n^{2} \pi^{2}\right)}\left[1-\frac{2 k^{\prime}}{n \pi s_{n}}\left(c_{n}-e^{-2 k h}\right) \zeta_{n}\right. \\
\left.+\cos (2 f)+(-1)^{n+1} \cos 2\left(f-k^{\prime}\right)\right]
\end{array}
$$

where

$$
s_{n}=\sinh \left(\lambda_{n} h\right), \quad c_{n}=\cosh \left(\lambda_{n} h\right) \quad \zeta_{n}=\frac{1}{2}\left(1-(-1)^{n}\right), \quad k^{\prime}=k L,
$$

and $\mathcal{N}=\sigma B_{0}^{2} /(\rho \dot{f})$ is the (dimensionless) magnetic interaction parameter which measures the ratio of magnetic forces to inertia. Typically this parameter will be small. For example, taking $f(t)=\omega t$ where $\omega$ is a constant, represents a periodic field travelling with velocity $\omega / k$. The evolution equation for each mode is a forced simple harmonic oscillator of the form,

$$
\ddot{a}_{n}+\Omega_{n}^{2} a_{n}=A_{n} \cos (2 \omega t)+B_{n} \sin (2 \omega t)+C_{n}
$$

where $A_{n}, B_{n}$ and $C_{n}$ are constants. This equation can be solved by elementary methods; resonance occurs when $2 \omega=\Omega_{n}$, in which case the solutions grow linearly with time. Figure 3 shows an example of the freesurface displacement.

\subsection{Aluminium Reduction Cells}

Figure 3 shows a diagram of an aluminium reduction cell. Aluminium is produced by reducing aluminium oxide electrolytically. To lower the melting point $\mathrm{Al}_{2} \mathrm{O}_{3}$ is dissolved in a mixture of sodium and aluminium fluorides to produce cryolite which forms the first fluid layer. An intense electric current passes through this layer via the carbon anode, the electrolysis producing molten aluminium which accumulates in the layer below.

The intense currents and associated magnetic fields give rise to a body force which can destabilise the interface between the cryolite and aluminium layers. Since cryolite is by far the poorest electrical conductor, a displacement of this interface will divert electric current to flow preferentially through the narrower sections of the layer (figure 5), causing a change in the Lorentz force field $\mathbf{J} \times \mathbf{B}$ which may tend to enhance the displacement. As usual the interface displacement $\eta$ is expanded in the form (2). In the absence of electromagnetic forces the evolution of the coefficients $a_{n}(t)$ would be simply described by decoupled harmonic oscillators,

$$
\ddot{a}_{n}+\Omega_{n}^{2} a_{n}=0, \quad n=1,2, \ldots,
$$




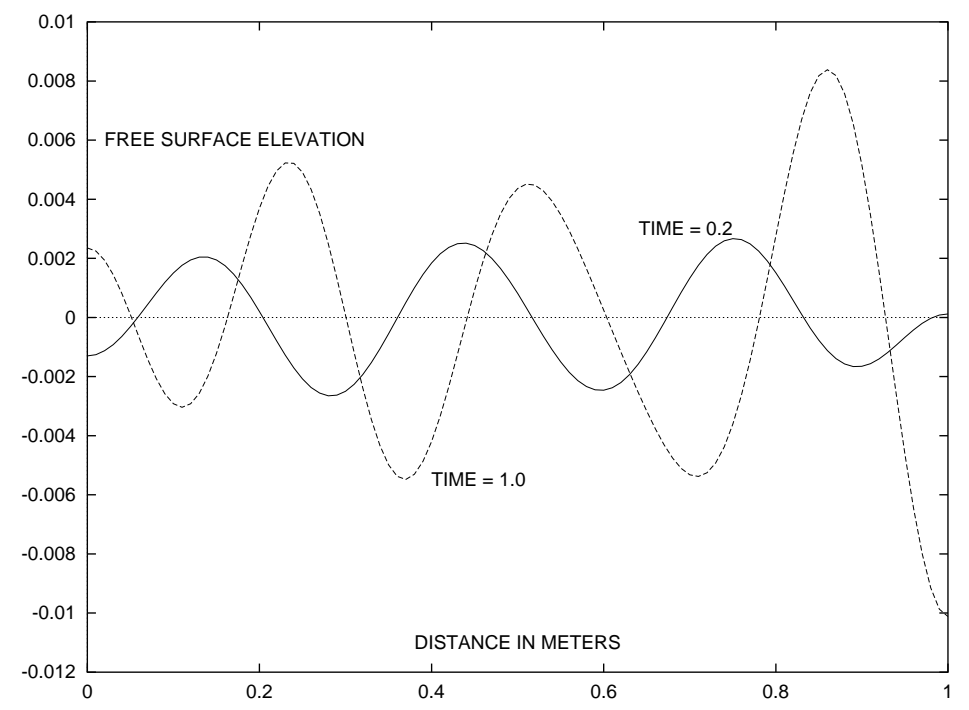

Figure 3. Surface wave excited in a tank of mercury. The tank length is $1 \mathrm{~m}$ and the depth $20 \mathrm{~cm}$. Other parameters are: $k=10, \omega=10 \mathcal{N}=0.1$.

where the $\Omega_{n}$ are the frequencies of the normal irrotational wave modes. We assume that in the presence of a uniform current (figure 5a) the interface

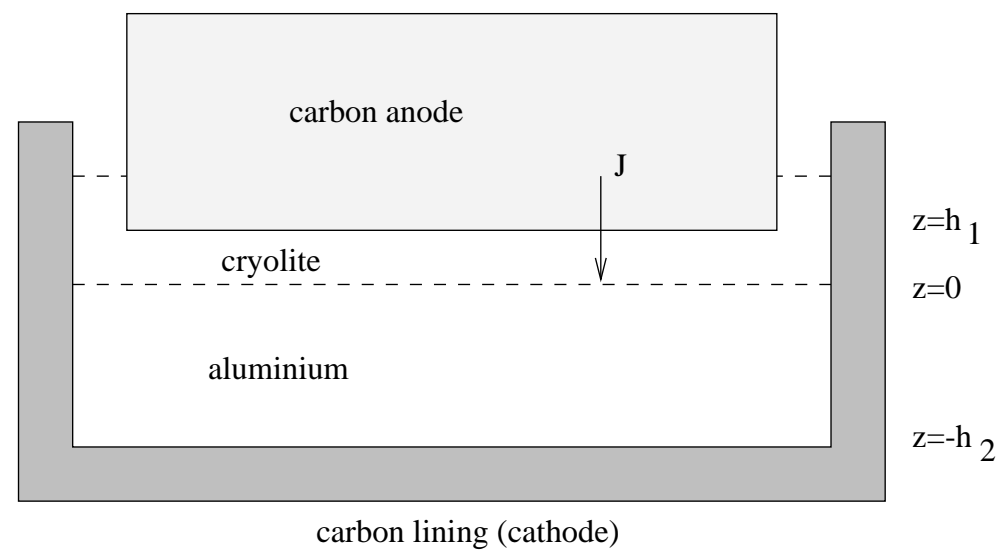

Figure 4. Diagram of an aluminium reduction cell 


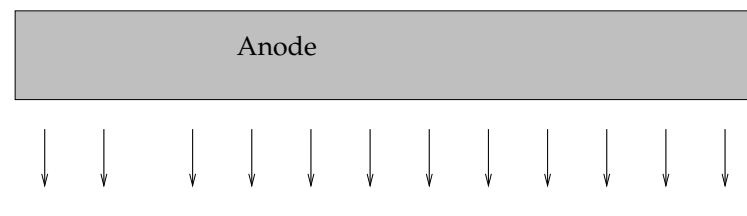

Cryolite

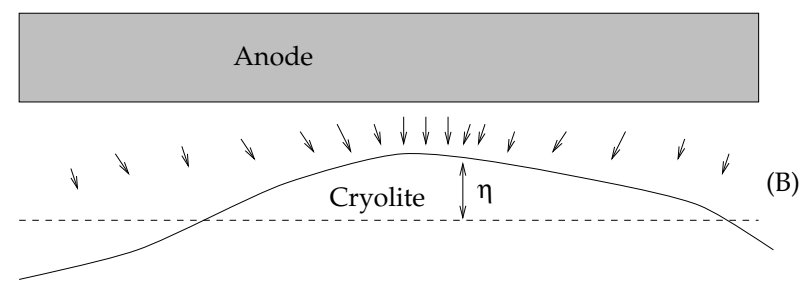

Figure 5. Current flow through the cryolite. In (A) the cryolite/aluminium interface is flat and the current uniform. In (B) the current flows preferentially through the narrowest part of the cryolite layer.

remains plane, so no forcing term appears in the above evolution equations (the electromagnetic forces being balanced by pressure). In a linear theory the displacement $\eta$ of the interface will produce a $\mathbf{J} \times \mathbf{B}$ body force which is linearly related to $\eta$, so that we can write,

$$
\mathbf{J} \times \mathbf{B}=\sum_{n=1}^{\infty} a_{n} \mathbf{f}_{n}
$$

where $\mathbf{f}_{n}$ is the body force due to a displacement $\eta=E_{n}$ of the free surface. Equation (12) now takes the form,

$$
\ddot{a}_{n}+\Omega_{n}^{2} a_{n}=\sum_{m=0}^{\infty} \beta_{n m} a_{m}(t)=0, \quad \beta_{n m}=\frac{\lambda_{n} \mathcal{T}_{n}}{\rho\left\|E_{n}\right\|^{2}}\left\langle\mathbf{f}_{n} \cdot \nabla \phi_{n}\right\rangle .
$$

The calculation of the $\beta_{n m}$ coefficients is straightforward, and details for a simple model cell are given in [6]. However the form of the equations (16) is independent of the detailed structure of the electromagnetic fields and other complicated features of a typical cell.

Even without detailed information on the $\beta_{n m}$ coefficients we can deduce certain important features. First, the waves no longer evolve independently, and a single given mode may excite waves of any other mode via the $\beta_{m n}$ coupling. Also if the $\beta_{m n}$ are small (which is typically the case) it can be 
shown that the system is most easily destabilised if two natural frequencies lie close together. The system can then be analysed approximately taking account of those two modes only [6].

\section{Conclusions}

The essence of our method is to expand the liquid free surface in terms of eigenmodes, and use an inner product method to derive evolution equations for each mode. One advantage of this method is that it easily deals with rotational body forces without requiring detailed knowledge of the rotational flow.

Here only a linearised analysis has been considered, but the method can be extended to give nonlinear evolution equations for the second-order free surface perturbation [4].

Viscosity is quite easily included, bringing an additional term

$$
-\nu \int_{V}(\nabla \times \boldsymbol{\omega}) \cdot \nabla \phi \mathrm{d} V=\int_{S_{W}+S_{B}}(\nabla \phi \times \boldsymbol{\omega}) \cdot \mathrm{d} \mathbf{S},
$$

to the evolution equation. Here $\boldsymbol{\omega}=\nabla \times \mathbf{v}$ is the vorticity, which at high Reynolds number is confined to boundary layers on the walls and can be estimated by boundary-layer analysis.

\section{References}

1. T. B. Benjamin and F. Ursell. The stability of the plane free surface of a liquid in vertical periodic motion. Proc. R. Soc. Lond., A225:505-515, 1954.

2. M. Faraday. Phil. Trans. 319.

3. L. M. Hocking, The damping of capillary-gravity waves at a rigid boundary. $J$. Fluid Mech. 179:253-266, 1987.

4. R. Kinsella. Second-order evolution equations and solutions for waves in a tank MCMS dissertation, University of Waikato 1998.

5. N. W. McLachlan. Theory and application of Mathieu functions. Oxford, Clarendon Press, 1947.

6. A. D. Sneyd and A. Wang, Interfacial instability due to MHD mode coupling in aluminium reduction cells. J. Fluid Mech. 263:343-359, 1994. 


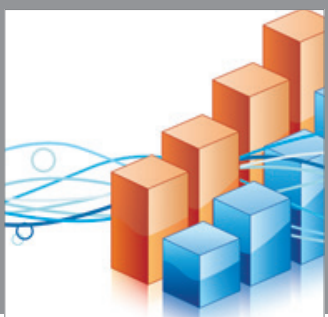

Advances in

Operations Research

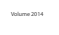

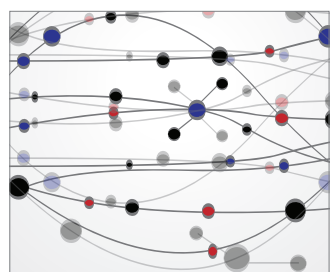

\section{The Scientific} World Journal
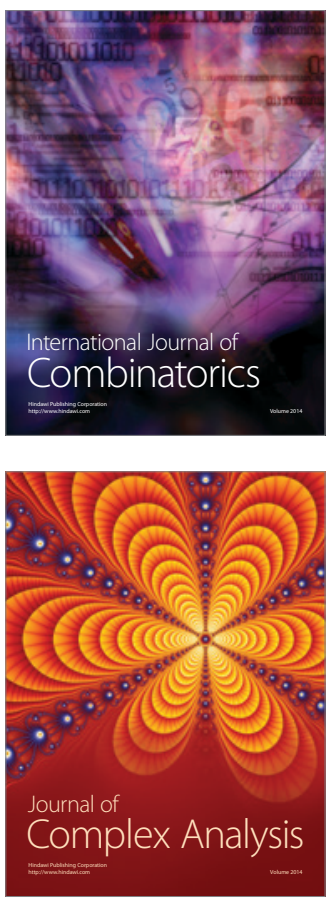

International Journal of

Mathematics and

Mathematical

Sciences
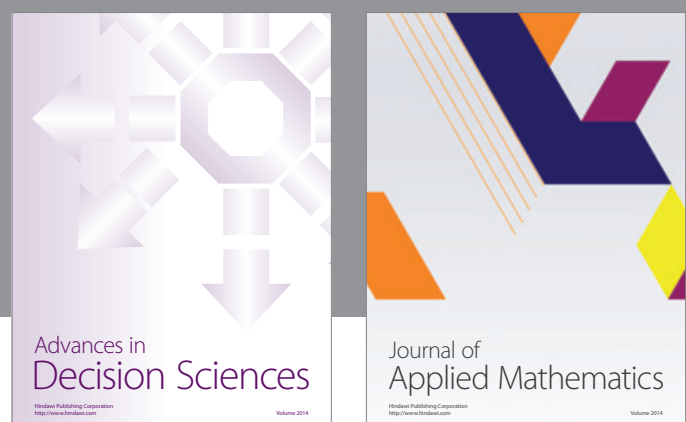

Journal of

Applied Mathematics
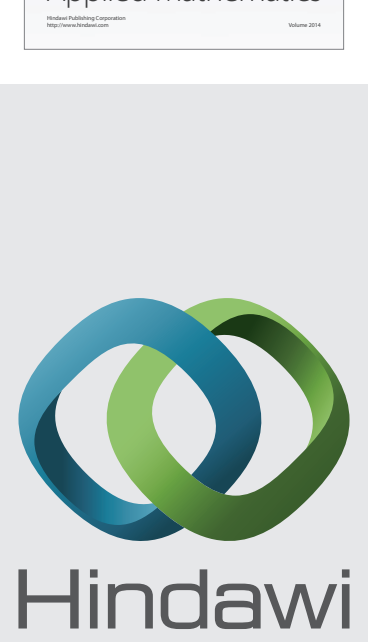

Submit your manuscripts at http://www.hindawi.com
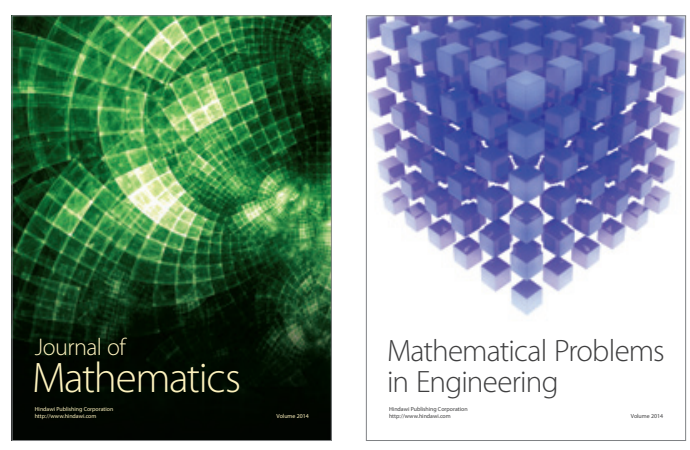

Mathematical Problems in Engineering
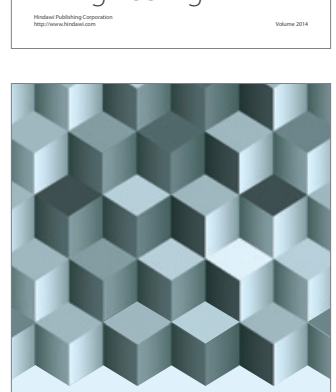

Journal of

Function Spaces
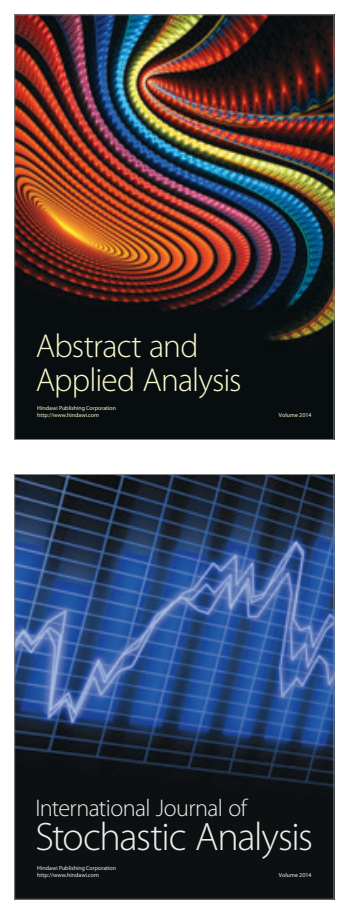

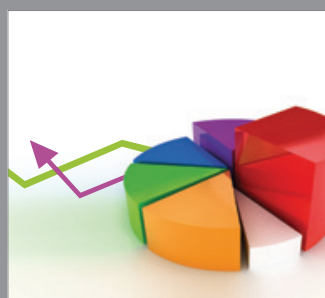

ournal of

Probability and Statistics

Promensencen
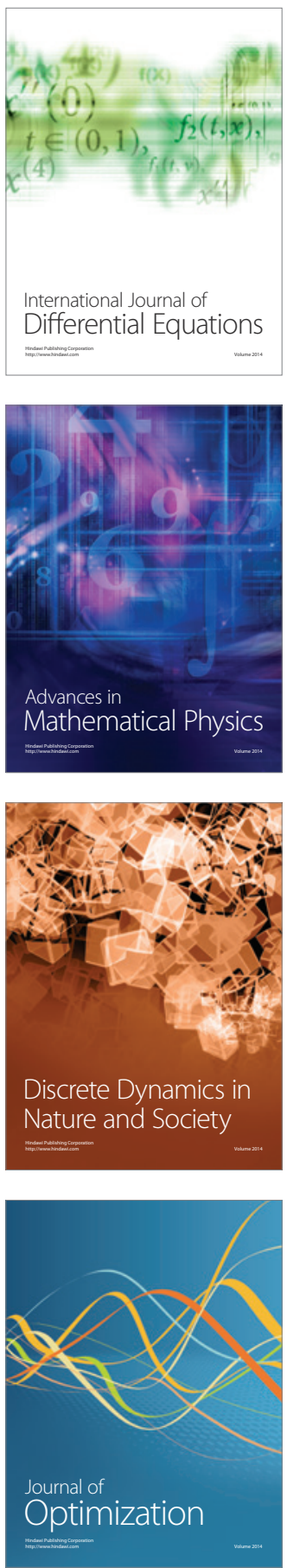